breast disease, lobular carcinoma, patients undergoing breast cancer surgery with $2 \mathrm{CPT}$ codes with ambiguous category placement and septic patients at time of surgery. For each intervention, a total of 16 complications were clustered into 8 groups and examined over the 13-year period. ALN management was categorized as follows: no intervention on ALNs, or ALN surgery (SLNB or ALN dissection (ALND)). Chi-square tests were performed for demographic and complication rate analysis. Smoothed linear regression and non-parametric Mann- Kendall test assessed complication trends. Uni-variate and multivariate logistical regression were computed to associate odd's ratio for comorbidities, surgical predictors and patients demographics. RESULTS/ANTICIPATED RESULTS: A total of 226,899 patients met the inclusion criteria. Annual breast surgery trends changed as follows: PM $45.6 \%$ to 45.9 ( $\mathrm{p}=0.21$ ), M $36.8 \%$ to $25.5 \%(\mathrm{p}=0.001), \mathrm{M}+\mathrm{R} 15.7 \%$ to $23.6 \%(\mathrm{p}=0.03)$ and $\mathrm{OS} 1.8 \%$ to $5.0 \%(\mathrm{p}=0.001)$. Analyzing the patient cohort who underwent breast conservation, categorical analysis showed a decreased use of PM alone ( $96 \%$ to $90 \%$ ) with an increased use of OS (4\% to $10 \%)$. For the patient cohort undergoing mastectomy, $M$ alone decreased (69\% to $52 \%$ ); $\mathrm{M}+\mathrm{R}$ with muscular flap decreased ( $9 \%$ to $2 \%$ ); and $\mathrm{M}+\mathrm{R}$ with implant placement increased (20\% to $41 \%)$ - all 3 trends $\mathrm{p}<0.0001$. The rate of ALN management has changed as follows: SNLB or ALND significantly increased in mastectomy patients from $53.6 \%$ to $69.5 \%$ (SS $1.5 \%, \mathrm{R} 20.69, \mathrm{p}<0.01$ ), while it changed little in the BCS population: $22.5 \%$ to $26.4 \%$ (SS $0.4 \%$, R2 $0.18, \mathrm{p}=0.09$ ). Complication rates have steadily increased in all mastectomy groups $(\mathrm{p}<0.05)$ but not in BCT. Cumulative complication rates between surgical categories were significantly different in each complication cluster (all $\mathrm{p}<0.0001$ ). Overall complication rates were: PM: $2.25 \%$, OS: $3.2 \%$, M: $6.56 \%, \mathrm{M}+\mathrm{MF}: 13.04 \%$ and $\mathrm{M}+\mathrm{I}: 5.68 \%$. The most common predictive risk factors were mastectomy interventions, increasing operative time, ASA class and BMI, smoking, recent weight loss, history of CHF, COPD and bleeding disorders (all $\mathrm{p}<0.001$ ). Patients who were non-diabetic, younger $(<60)$ and treated as outpatient all had protective OR for an acute complication $(\mathrm{p}<0.0001)$. DISCUSSION/SIGNIFICANCE OF IMPACT: The modern era of breast surgery is identified by the increasing use of reconstruction for patients undergoing breast conservation (in the form of OS) and mastectomy (in the form of $\mathrm{M}+\mathrm{R}$ ). Despite national recommendations for the management of axillary lymph nodes in patients undergoing breast surgery for DCIS, nearly $30 \%$ of cases continue to be mismanaged: more than $30 \%$ of patients with DCIS undergoing mastectomy fail to receive SLNB, and more than $26 \%$ of DCIS patients undergoing BCS are still receiving axillary lymph node surgery. Our study provides data showing significant trends that will impact the future of both breast cancer surgery and breast training programs. We also provide data comparing nationwide acute complication rates following different breast cancer surgeries that can be used to inform patients during surgical decision making.

3164

Do cancer survivors understand their risk factors for recurrence and the value of coordinated care between an oncologist and a primary care physician? A survey of endometrial and cervical cancer patients

Subhjit Sekhon ${ }^{1}$, Lindsay Kuroki ${ }^{1}$ and Graham Colditz ${ }^{1}$

${ }^{1}$ Washington University in St. Louis, Institute Of Clinical and Translational Sciences

OBJECTIVES/SPECIFIC AIMS: To evaluate gaps in knowledge for women who are cancer survivors regarding the impact of comorbidities and lifestyle behaviors on endometrial and cervical cancer risk, and to assess prevalence of established care with a primary care physician (PCP) among patients and evaluate acceptability of referral to a PCP METHODS/STUDY POPULATION: Single institution cross-sectional study examining all women aged 18 or older with a diagnosis of cervical or endometrial cancer who present for care by a gynecologic oncologist at Barnes-Jewish Hospital/ Washington University in St. Louis School of Medicine. Patients will be invited to complete a survey specific to cancer diagnosis that includes questions on participant background and sociodemographic information, knowledge of risk factors for their specific cancer site, and whether or not the patient has a primary care provider and the acceptability of referring RESULTS/ANTICIPATED RESULTS: Majority of women will be unaware of how comorbidities affect cancer risk and treatment outcomes. For women without a PCP, we anticipate that they will be accepting towards the notion of being referred to one for establishing care. DISCUSSION/ SIGNIFICANCE OF IMPACT: Pilot information from this study will 1 . Allow providers to improve cancer survivorship care plans by increasing collaboration between PCPs and oncologists to provide ongoing care, and 2. Afford information for providers on where gaps in knowledge exist so as to better education patients.

3038

\section{Examining the association between inpatient opioid prescribing and patient satisfaction.}

Olena Mazurenko ${ }^{1}$, Justin Blackburn, Matthew Bair, Areeba Kara and Christopher A. Harle

${ }^{1}$ Indiana University School of Medicine

OBJECTIVES/SPECIFIC AIMS: Research overview: Providing patient-centered care is increasingly a top priority in the U.S. healthcare system.1,2 Hospitals are required to publicly report patient-centered assessments, including results from the Hospital Consumer Assessment of Healthcare Providers and Systems (HCAHPS) patient satisfaction surveys.3 Furthermore, clinician and hospital reimbursements are partially determined by performance on patient satisfaction measures. 3 Consequently, hospitals and clinicians may be incentivized to improve patient satisfaction scores over other important outcomes.4 Paradoxically then, the pursuit of patient-centered care may lead clinicians to fulfill patient requests for unnecessary and potentially harmful treatments.5 Opioid prescribing during hospitalizations may be particularly affected by clinicians' seeking to optimize patient satisfaction scores.6,7 Satisfaction with pain care is an important predictor of overall patient satisfaction in the HCAHPS surveys, 8,9 and clinicians report increased pressure to fulfill patient requests for immediate pain-relief.10,11 Therefore, clinicians may prescribe opioids to avoid receiving lower patient satisfaction scores.12,13 Furthermore, clinicians lack clear guidance on opioid prescribing for some populations, including non-surgical inpatients, who represent almost half of all hospitalizations.14 To reduce clinicians' incentive to prescribe opioids as a means of achieving patient satisfaction, the Center for Medicare and Medicaid Services (CMS) temporarily removed questions related to patient satisfaction with pain care from the clinician and hospital reimbursement formulas beginning in 2018.15 Importantly, prior research1620 has not rigorously tested the hypothesis implied by the CMS policy change: that certain opioid prescribing practices in inpatient pain care are associated with higher patient satisfaction. Objectives: The purpose of this study was to evaluate the association between the receipt/dose of opioids during non-surgical hospitalizations and 
patient satisfaction measured by the HCAHPS survey. METHODS/ STUDY POPULATION: Methods/Study Population: We conducted a pooled cross-sectional study of adults (18 and older) with non-surgical hospitalizations within the 11-hospital healthcare system in a Midwestern state from 2011-2016. Data were extracted from electronic health records and linked to HCAHPS patient satisfaction surveys. We estimated the propensity score for receipt of any opioids during hospitalization and separately the receipt of high dose opioids ( $\geq 100$ morphine milligram equivalent [MME]) based on patient, encounter, and facility characteristics for all hospitalizations with complete data. We used nearest neighbor matching to construct two matched samples to minimize selection bias and confounding by indication. We used a standardized difference threshold of $<$ 0.1 as an indication of the balance between matched groups. Outcomes were compared with a test on the equality of proportions using large-sample statistics. All analysis was performed in STATA 14.0 analytical software. Main outcomes: We analyzed four dependent variables. Two pain-specific patient satisfaction variables were derived from the responses to the following survey questions: 1) "During this hospital stay, how often your pain was well controlled? (pain control)" and 2) "During this hospital stay, how often did the hospital staff do everything they could to help you with your pain? (pain help)", with 4-point Likert scale responses ranging from "Never" to "Always." We also used two global satisfaction measures derived from the responses to the following survey questions: 1) "Using any number from 0 to 10, where 0 is the worst hospital possible and 10 is the best hospital possible, what number would you use to rate this hospital during your stay (overall patient satisfaction)?" and 2) "Would you recommend this hospital to your friends and family (willingness to recommend a hospital)? (4-point scale of "Definitely Yes" to "Definitely No"). Because the responses are not normally distributed, and the response options are truncated, we dichotomized each of these questions following previously published approaches8 and CMS methodology3 (e.g. "always" vs. all other responses or "9 or 10 rating" vs. all others). RESULTS/ ANTICIPATED RESULTS: Results: Among 17,691 patients who reported that they needed pain medications during hospitalization in their HCAHPS survey, $43.7 \%(n=7,735)$ received opioids. Among the matched sample $(n=8,848), 55 \%$ were female, $90 \%$ were white, $9 \%$ were black, $74 \%$ were emergency admissions, $29 \%$ had a circulatory diagnosis, $92 \%$ were discharged home, and the average pain score ranged from 0.2 to 7.1 during the hospital stay. Compared to matched patients hospitalized but did not receiving opioids, those who received opioids did not significantly differ in their rating of pain help ( $75 \%$ of patients without opioids rated that they always received help for their pain versus $75 \%$ of patients with opioids; $\mathrm{p}=.78$ ), pain control (55\% of patients without opioids reported that their pain was well controlled versus $54 \%$ on opioids; $\mathrm{p}=.93$ ), willingness to recommend the hospital (69\% of patients without opioids reported that they would definitely recommend a hospital versus $71 \%$ with opioids; $\mathrm{p}=.16$ ) and overall rating of their care ( $47 \%$ of patients without opioids rated their hospitalization as 10 versus $46 \%$ on opioids; $\mathrm{p}=.22$ ). DISCUSSION/SIGNIFICANCE OF IMPACT: Discussion: We found no evidence that receipt of opioids is associated with patient satisfaction, including at doses. To our knowledge, this is the first study that used propensity score matching to examine the association between inpatient opioid prescribing practices and patient satisfaction. Furthermore, our sample is unique in the inclusion of patients hospitalized for non-surgical indicators over a five year period in the multi-hospital healthcare system in a Midwestern state. Our findings add to the existing literature which has shown contradictory associations between opioid prescribing and patient satisfaction.16-22 Specifically, few studies that looked at surgical inpatients showed a lack of association between patient satisfaction 16,18 and opioid prescribing, whereas others showed that receipt of opioids was associated with lower patient satisfaction.1720 Our findings may imply that satisfaction with pain care may be achieved without administering opioids to non-surgical inpatients. Alternatively, satisfaction with pain care may not be influenced by opioid prescribing for non-surgical inpatients. Future research should further examine the association between opioid prescribing and patient satisfaction among non-surgical inpatients on a national scale to get a better understanding of the relationship between certain pain care practices and patient satisfaction.

3014

Identification of a Cohort to Study Treatment Patterns in Elderly Patients with Incident Hodgkin Lymphoma (HL) using Surveillance, Epidemiology and End Results (SEER)-Medicare Data

Angie Mae Rodday ${ }^{1}$, Theresa Hahn, Peter Lindenauer and

Susan Parsons

${ }^{1}$ Tufts University

OBJECTIVES/SPECIFIC AIMS: (1) To define and describe a cohort of patients aged $\geq 65$ years with incident HL from SEER-Medicare data. (2) To identify patient, disease, and system-level factors associated with initial treatment for HL. METHODS/STUDY POPULATION: This retrospective cohort study utilized SEERMedicare data from 1999-2014. Patients with incident classical HL were identified using SEER registry histology groupings. The cohort was restricted to those with Medicare Part A and B fee-for-service for 3 months prior to and 1 year after diagnosis (or until date of death) in order to fully capture claims for outpatient chemotherapy. Patients were excluded for the following reasons: missing month of HL diagnosis; unknown diagnostic confirmation; reporting from autopsy or death certificate; or another cancer diagnosis $+/-2$ years of the HL diagnosis. Demographic and disease characteristics were defined based on SEER registry data. Broad treatment categories were defined using SEER data, while detailed treatment categories will be defined based on Medicare claims. Length of follow-up was defined as the number of months until the earliest of the following: death; end of continuous Medicare Part A and B fee-for-service enrollment; or the end of the available data (12/31/2014). Demographic, disease, and preliminary treatment characteristics were described for the cohort. Future analyses will explore patient and disease factors, including comorbidities and an estimate of frailty, as well as system-level factors associated with initial treatment of HL. RESULTS/ANTICIPATED RESULTS: We identified 2909 patients meeting eligibility for the cohort. The median length of follow-up was 22 months $(\mathrm{Q} 1=5, \mathrm{Q} 3=62)$. Median age was 75.9 years $(\mathrm{Q} 1=70$, $\mathrm{Q} 3=81$ ), $49.6 \%$ were female, and $82.6 \%$ were non-Hispanic/White. Only $11.5 \%$ of patients were in rural or non-urban areas. $13.8 \%$ of patients were dual eligible for both Medicare and Medicaid. Nodular sclerosis was the most common histology (35.2\%), followed by mixed cellularity $(21.1 \%) ; 36.5 \%$ had histology that was not otherwise specified. Patients were evenly distributed across Ann Arbor Stage (21.8\% with I; $22.3 \%$ with II; $25.8 \%$ with III; $24.2 \%$ with IV; $6 \%$ unknown). B symptoms were present in $35.2 \%$ of patients, absent in $39.6 \%$, and unknown in $25.2 \%$. Neither tumor bulk nor international prognostic score were available via SEER registry data. According to SEER registry data, most patients received some treatment for their HL (81.9\%) and $75 \%$ of those patients initiated treatment within one 